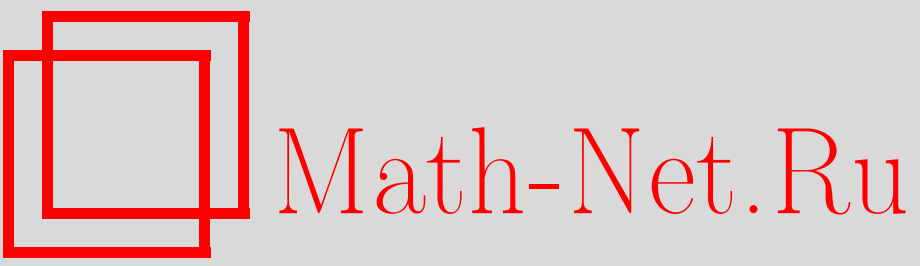

В. М. Бухштабер, О. В. Карпов, С. И. Тертычный, О свойствах дифференциального уравнения, описывающего динамику сильношунтированного перехода Джозефсона, УМН, 2004, том 59, выпуск 2, 187-188

DOI: https://doi.org/10.4213/rm725

Использование Общероссийского математического портала Math-Net.Ru подразумевает, что вы прочитали и согласны с пользовательским соглашением

http://www.mathnet.ru/rus/agreement

Параметры загрузки:

IP : 54.164 .48 .24

26 апреля 2023 г., 15:25:42 


\title{
О СВОЙСТВАХ ДИФФЕРЕНЦИАЛЬНОГО УРАВНЕНИЯ, ОПИСЫВАЮШЕГО ДИНАМИКУ СИЛЬНОШУНТИРОВАННОГО ПЕРЕХОДА ДЖОЗЕФСОНА
}

\author{
В. М. БУХШТАБЕР, О.В. КАРПОВ, С.И. ТЕРТЫЧНЫЙ
}

Уравнение вида

$$
\dot{\varphi}(t)+\sin \varphi(t)+q(t)=0
$$

успешно используется (см. [1]-[4]) для описания свойств сильношунтированных переходов Джозефсона в сверхпроводниках. Здесь $\varphi(t)$ имеет смысл разности фаз параметра порядка (макроскопической волновой функции, описывающей коллективные свойства “жидкости” куперовских пар электронов в сверхпроводнике),$-q(t)$ описывает ток, подводимый из внешней цепи к переходу. Физика вопроса приводит к следующей математической задаче.

Дана функция $q=q(t ; u)$, которая зависит от вектора параметров $u=\left(u_{1}, \ldots, u_{n}\right)$ и является периодической по $t$ с периодом $T=u_{n}$. Найти область $U \subset \mathbb{R}^{n}$ такую, что для $u \in U$ все решения уравнения (1) обладают свойством:

$$
\lim _{n \rightarrow \infty}(\varphi(t+(n+1) T)-\varphi(t+n T))=2 \pi k
$$

для некоторого общего целого $k$, назьваемого порядком ступеньки Шапиро.

Наш подход к этой задаче опирается на следующий общий результат.

Лемма 1. Пусть дана вещественная функция $q(t)$. Функции $\varphi(t)$ и $\xi(t)$ дают решение системь

$$
\begin{aligned}
& \dot{\varphi}(t)+\sin \varphi(t)+q(t)=0, \\
& \dot{\xi}(t)-\cos \varphi(t)=0
\end{aligned}
$$

с $\varphi(0)=\varphi_{0}, \xi(0)=0$ тогда и только тогда, когда функции $x(t), y(t)$ вида

$$
\begin{aligned}
& x(t)=\exp (\xi(t) / 2) \cos \left(\left(\varphi(t)-\varphi_{0}\right) / 2\right), \\
& y(t)=\exp (\xi(t) / 2) \sin \left(\left(\varphi(t)-\varphi_{0}\right) / 2\right)
\end{aligned}
$$

дают решение системь

$$
\begin{aligned}
2 \dot{x} & =\left(\cos \varphi_{0}\right) x+\left(q-\sin \varphi_{0}\right) y, \\
-2 \dot{y} & =\left(\sin \varphi_{0}+q\right) x+\left(\cos \varphi_{0}\right) y
\end{aligned}
$$

$c x(0)=1, y(0)=0$.

Положим $\chi(t)=\frac{x+j y}{x-j y}$, где $j=\sqrt{-1}$. Имеем $\chi(t)=\exp j\left(\varphi(t)-\varphi_{0}\right)$. Таким образом, решение $\varphi(t)$ уравнения (1) удовлетворяет условию (2) тогда и только тогда, когда последовательность функций $\chi_{n}(t)=\chi(t+n T), n=0,1,2, \ldots$, поточечно сходится.

Непосредственная проверка показывает, что если $x_{1}(t), y_{1}(t)$ - решение системы $(5)$ с $x_{1}(0)=$ $1, y_{1}(0)=0$, то функции

$$
x_{2}(t)=\eta_{1}(t) x_{1}(t) \text { и } y_{2}(t)=\eta_{1}(t) y_{1}(t)+\eta_{2}(t),
$$

где

$$
\eta_{1}(t)=\int_{0}^{t} \frac{q(\tau)-\sin \varphi_{0}}{x_{1}(\tau)^{2}} d \tau \text { и } \eta_{2}(t)=\frac{1}{x_{1}(t)}
$$

представляют собой решение системы (5) с $x_{2}(0)=0, y_{2}(0)=1$.

Работа выполнена при финансовой поддержке Российского фонда фундаментальных исследований (грант № 03-01-00406). 
Положим $\mathbf{X}(t)=\left(\begin{array}{ll}x_{1}(t) & x_{2}(t) \\ y_{1}(t) & y_{2}(t)\end{array}\right)$ и $\mathbf{X}_{n}(t)=\mathbf{X}(t+n T), n=0,1,2, \ldots$ Пусть теперь $q(t)-$ периодическая функция с периодом $T$. Из общей теории систем линейных обыкновенных дифференциальных уравнений с периодическими коэффициентами следует, что если $\mathbf{X}(0)=\mathbf{I}$ (единичная матрица), то

$$
\mathbf{X}_{n}(t)=\mathbf{X}(T)^{n} \mathbf{X}(t), \quad n=0,1,2, \ldots .
$$

Отметим, что $\operatorname{det} \mathbf{X}(t)=1$. Имеет место следующий общий результат.

Лемма 2. Пусть $\mathbf{A}=\left(a_{i k}\right)$ - вещественная $(2 \times 2)$-матрица и $\gamma_{1}, \gamma_{2}-$ ее собственньее числа. Положим $a_{11}+a_{22}=2 a$. Пусть $\operatorname{det} \mathbf{A}=1$. Тогда: если $\gamma_{1}=\gamma_{2}=\gamma$, то $\gamma=a$ u $a^{2}=1 ;$ eсли $\gamma_{1} \neq \gamma_{2}$, mo $\gamma_{1}=\gamma=a+\sqrt{a^{2}-1}, \gamma_{2}=\gamma^{-1}=a-\sqrt{a^{2}-1}$, гдe $|a| \neq 1$. Более того, $|\gamma| \neq 1$ тогда и только тогда, когда $|a|>1$.

Пусть теперь $\mathbf{A}=\mathbf{X}(T)$. Тогда из лемм 1 и 2 получаем следующее утверждение.

Теорема 1. Решения уравнения (1) c $q(t ; u)$ при данном и удовлетворяют условию (2) тогда и только тогда, когда $|a|>1$, где $2 a=\left(x(T)+x(T)^{-1}+\eta_{1}(T) y(T)\right)$, и в этом случае

$$
\begin{aligned}
& \exp j\left(\varphi(t+n T)-\varphi_{0}\right)=\frac{\alpha_{n} x(t)+\beta_{n} y(t)}{\bar{\alpha}_{n} x(t)+\bar{\beta}_{n} y(t)}, \\
& \text { əде } \alpha_{n}=c_{11} \gamma^{n}+c_{12} \gamma^{-n}, \beta_{n}=c_{22} \gamma^{n}+c_{21} \gamma^{-n} u \\
& c_{11}=a_{21}\left[a_{12}-j\left(a_{11}-\gamma\right)\right], \quad c_{12}=-\left(a_{11}-\gamma\right)\left[\left(a_{22}-\gamma^{-1}\right)-j a_{21}\right], \\
& c_{21}=\left(a_{22}-\gamma^{-1}\right)\left[a_{12}-j\left(a_{11}-\gamma\right)\right], \quad c_{22}=-a_{12}\left[\left(a_{22}-\gamma^{-1}\right)-j a_{21}\right] . \\
& \frac{c_{11} x(t)+c_{22} y(t)}{\bar{c}_{11} x(t)+\bar{c}_{22} y(t)} \text { npu }|\gamma|>1 \quad u \quad \frac{c_{12} x(t)+c_{21} y(t)}{\bar{c}_{12} x(t)+\bar{c}_{21} y(t)} \text { npu }|\gamma|<1 .
\end{aligned}
$$

ПРИМЕР 1. Пусть $-q(t ; u)=\iota_{0}+2 \pi \iota_{1} \sum_{n=0}^{\infty}\left[\delta\left(t-n T-\frac{1}{2} T-\frac{1}{2} \tau\right)-\delta\left(t-n T-\frac{1}{2} T+\frac{1}{2} \tau\right)\right]$, где $\delta(\cdot)$ - функция Дирака (см. [3], [4]). Тогда $u=\left(\iota_{0}, \iota_{1}, \tau, T\right)$ и область $U \subset \mathbb{R}^{4}$ задается неравенством

$$
\left|\cosh \left(\frac{T}{2} \sqrt{1-\iota_{0}^{2}}\right)-\frac{\sin ^{2}\left(\pi \iota_{1}\right)}{1-\iota_{0}^{2}}\left[\cosh \left(\frac{T}{2} \sqrt{1-\iota_{0}^{2}}\right)-\cosh \left(\left(\frac{T}{2}-\tau\right) \sqrt{1-\iota_{0}^{2}}\right)\right]\right|>1
$$

\section{СПИСОК ЛИТЕРАТУРЫ}

[1] К. К. Лихарев, Б. Е. Ульрих. Системы с джозефсоновскими контактами. М.: Изд-во МГУ, 1978. [2] А. Бароне, Дж. Патерно. Эффект Джозефсона. Физика и применение. М.: Мир, 1984. [3] В. М. Бухштабер, О. В. Карпов, С. И. Тертычный // ЖЭТФ. 2001. Т. 120. №6 (12). С. 1478-1485. [4] В. М. Бухштабер, О. В. Карпов, С. И. Тертычный // ЖКЭТФ. 2003. Т. 124. №3 (9). С. 691-699.

ВНИИ Физико-технических и радиотехнических измерений, Менделеево, Московская область
Принято редколлегией 23.03 .2004 